\title{
Desenvolvimento de Atividades Convectivas Sobre a Região Nordeste do Brasil, Organizada Pela Extremidade Frontal
}

\author{
Maicon Eirólico Veber ${ }^{1,2}$ (D), Natalia Fedorova ${ }^{2}$ (D), Vladimir Levit ${ }^{2}$ \\ ${ }^{I}$ Instituto Nacional de Pesquisas Espaciais, Cachoeira Paulista, SP, Brasil. \\ ${ }^{2}$ Instituto de Ciências Atmosféricas, Universidade Federal de Alagoas, Maceió, AL, Brasil.
}

Recebido em: 12 de Julho de 2020 - Aceito em: 29 de Dezembro de 2020

\begin{abstract}
Resumo
Nas extremidades de zonas frontais que atingem o Nordeste brasileiro (NEB), em alguns eventos, desenvolve-se convecção, e em outras frentes isso não ocorre. Os eventos em que ocorre convecção profunda sobre o NEB, estão associados a eventos de precipitação intensa e possíveis transtornos associados. Por isso, o objetivo geral é analisar as diferenças dos processos dinâmicos e termodinâmicos nestes eventos e obter os padrões de circulação. Foram utilizados dados de reanálise do modelo global do NCEP/NCAR e imagens de satélite do canal infravermelho para o período de dez anos (2000-2009). Durante este período, foram identificadas 103 zonas frontais no NEB. O maior número das frentes foi detectado nos anos de 2002 e 2007 (14 e 13, respectivamente) e o menor, nos anos de 2004 e 2005 (5 e 6 , respectivamente). A atividade convectiva na extremidade frontal foi formada em 68 eventos (Grupo 1, G1), e em 35 eventos foi identificada banda de nebulosidade pouco ativa (Grupo 2, G2). A análise termodinâmica foi baseada na análise de dados de radiossondagens em 5 estações do NEB e a instabilidade foi avaliada pela Convective Available Potencial Energy (CAPE) e índices de instabilidade K, Total Totals (TT) e Lifted index (LI). CAPE e LI mostraram as maiores diferenças entre os casos de G1 e G2. Os eventos do G1 foram encontrados durante todas as estações do ano, porém mais frequentes durante o verão (33 casos), nas transições fria (19 casos) e quente (14 eventos) e 1 evento no inverno. Os eventos do G2 foram mais frequentes durante o inverno (22 eventos), 7 eventos foram observados nas transições frio e quente e não foram observados durante o verão. Foram elaborados os padrões da circulação em G1 e G2 em baixos, médios e altos níveis. Sistemas sinóticos do G1 criaram confluência das correntes em baixos e médios níveis sobre o NEB, todavia no G2 foi observada circulação anticiclônica. Em altos níveis a presença do VCAN, ou de um cavado intenso, foi típico para G1.Os padrões de circulação e conclusões sobre utilização de índices de instabilidade pode facilitar na previsão de desenvolvimento de convecção na extremidade frontal.
\end{abstract}

Palavras-chave: sistemas frontais, processos convectivos, Nordeste do Brasil.

\section{Development of Convection on the Northeast Region of Brazil, organized by extremities of frontal zones.}

\begin{abstract}
At the extremities of frontal zones, which pass in the Northeastern Brazil (NEB), convection and intense rainfall in some events were developed and, in other fronts, were not. Therefore, the general objective is to analyze the differences in dynamic and thermodynamic processes in these events and to create circulation patterns. Reanalysis data from the global NCEP / NCAR model and satellite infrared images were used for the period of ten years (2000-2009). During the studied period, 103 frontal zones were identified in the NEB. The highest number of the fronts was detected in 2002 and 2007 (14 and 13, respectively) and the lowest in 2004 and 2005 (5 and 6, respectively). Convective activity at the front extremities was formed in 68 events (Group 1, G1) and in 35 events a low active cloud band was identified (Group 2, G2). The thermodynamic was based on the analysis of radiosonde data in the 5 meteorological stations of NEB and instability was evaluated by CAPE and instability indexes K, TT and LI. CAPE and LI showed the greatest differences in G1 and G2. G1 events were found during all seasons of the year, but, more frequently, they were detected during the
\end{abstract}

Autor de correspondência: Maicon Eirólico Veber, maicon.veber@gmail.com. 
summer (33 cases), in the cold (19 cases) and hot (14 events) transitions and 1 event in the winter. The G2 events were more frequent during the winter ( 22 events), 7 events were observed in the cold and hot transitions and none was observed during the summer. Circulation patterns in G1 and G2 were developed at the low, medium and high levels. Synoptic systems of G1 created confluence of currents at the low and medium levels over the NEB, however, in G2, an anticyclonic circulation was observed. The presence of Upper Tropospheric Cyclonic Vortex (UTCV) or an intense trough was typical for G1 at the high levels. Circulation patterns and conclusions about the use of instability indices can facilitate the forecast of the convection development at the frontal extremities.

Keywords: frontal systems, convective processes, Northeastern Brazil.

\section{Introdução}

Os Sistemas Frontais (SF) afetam as condições de tempo na América do Sul nas diferentes épocas do ano (Lemos e Calbete,1996; Quadro et al., 1996). Os SF geralmente são identificados facilmente por meio de imagens de satélites e preferencialmente se deslocam de sudoeste para nordeste sobre o continente e o oceano Atlântico adjacente (Oliveira, 1986). Por vezes, os SF alcançam latitudes próximas da região Tropical, por exemplo, oeste da Amazônia e ao longo da costa Nordeste do Brasil (NEB) (Cavalcanti e Kousky, 2009). Ainda segundo Cavalcanti e Kousky (2009), a máxima precipitação entre novembro e março no NEB está relacionada com a interação entre SF e a convecção tropical. Ao adentrarem a América do Sul, as frentes frias podem adquirir deslocamento para leste, modificando o tempo no sul do continente; ou para norte (nordeste), modificando as condições de tempo em todo o continente Sul-americano (Gemiack, 2005).

Quando esses sistemas se deslocam para norte (nordeste), chegam frequentemente até latitudes tropicais com intensidade considerável (Lemos e Calbete, 1996).

Kalnay (1996), Justi e Silva Dias (2002) e Cavalcanti e Kousky (2003), dentre outros, mostraram que a frequência de frentes frias diminui com o decréscimo da latitude e que suas passagens estão ligadas às mudanças de tempo nas regiões afetadas. Em latitudes mais altas, essas mudanças no tempo estão associadas com precipitação, queda de temperatura e mudança na direção e intensidade do vento. Em latitudes mais baixas, os efeitos das frentes estão relacionados principalmente com o aumento da precipitação.

Ao se deslocarem por latitudes tropicais, os SF podem interagir com a ZCIT e com o VCAN na costa do NEB, aumentando os processos convectivos (Cavalcanti e Kousky, 2009).

Oliveira (1986) realizou uma climatologia das frentes para o período de 1975 a 1984 e constatou que os SF podem interagir com a convecção local, especialmente durante a primavera e verão austral, quando os SF apresentam ampla penetração continental, uma condição necessária para essa interação.

Cruz (2008) realizou a determinação sinótica dos fatores que favorecem as influências frontais sobre o estado de Alagoas (AL). Nesse estudo, também constatou que as influências frontais sobre AL podem ser divididas em três grupos distintos que são: Grupo $1 \mathrm{em}$ que observou a pas- sagem da frente sobre o estado. Grupo 2 em que verificou a extremidade frontal sobre o Atlântico Tropical Sul, influenciando as condições do tempo sobre AL. Grupo 3 no qual a extremidade frontal sobre o Atlântico organizou atividades convectivas sobre $\mathrm{AL}$.

As zonas frontais, as quais atingem o NEB, mostram as características e estrutura modificada. Kousky (1979) utilizou para identificação das zonas frontais no sul da Bahia a temperatura virtual e potencial equivalente, pois nos campos de temperatura as frentes frias, por vezes, não são bem detectadas. Gemiacki (2005) mostrou que, nas zonas frontais na parte central do NEB, as variáveis de temperatura (média, máxima e mínima), temperatura potencial e umidade relativa foram pequenas, se comparadas com as do Sul e Sudeste. Todavia, nestas frentes foram observados a forte variação na velocidade e direção do vento, o aumento de pressão e as precipitações. Fedorova et al. (2016) incluíram na identificação das frentes no NEB a advecção de temperatura potencial equivalente e estabeleceram as variáveis as quais ajudam nesse processo. Estas variáveis são utilizadas neste trabalho e serão descritas na seção 2. O enfraquecimento dos gradientes de temperatura potencial, equivalente próximo à superfície, na região central do NEB, e a existência de mais gradientes mais intensos na camada $700-500 \mathrm{hPa}$, foi mostrada por Sinhory (2015).

Ao estudarem eventos extremos diários de chuva sobre o NEB e suas características atmosféricas, Brado Alves et al. (2017) evidenciaram em dias considerados chuvosos, entre fevereiro e maio, a incursão de uma massa de ar frio com anomalias negativas de temperatura de até $-5{ }^{\circ} \mathrm{C}$ sobre o NEB e sua vizinhança. Indicando, portanto, que as frentes frias forçam a ascensão do ar quente e úmido sobre a Região, sendo um fator físico essencial para instabilizar e provocar chuva intensa e homogênea sobre o NEB.

O objetivo deste estudo foi identificar a influência dos SF sobre o NEB, analisando o desenvolvimento de processos convectivos organizados pela extremidade frontal no NEB. Para alcançar os objetivos, necessitou-se identificar os fatores dinâmicos e termodinâmicos responsáveis pela formação desses processos convectivos. A identificação destes fatores e a criação de padrões sinóticos em níveis de padrões favorecerá a utilização dos resultados em ambiente operacional. Tendo, portanto, como intuito procurar melhorar a previsão desses eventos, que costumam provocar chuva intensa sobre o NEB. 


\section{Materiais e Métodos}

\subsection{Dados}

Todo estudo foi feito para o período de 10 anos, de 01 de janeiro de 2000 a 31 de dezembro de 2009. Foram utilizadas imagens dos satélites GOES e METEOSAT, no canal espectral infravermelho (canal 4 entre 10,2 e $11,2 \mu \mathrm{m}$ e $10,8 \mu \mathrm{m}$, respectivamente). Obtidas através do site do National Oceanic and Atmospheric Admistration (NOAA).

Foi utilizado um conjunto de dados para os quatro horários sinóticos padrões (00, 06, 12 e 18 UTC), das variáveis atmosféricas das reanálises do National Center for Environmental Prediction (NCEP) / National Center for Atmospheric Research (NCAR), com resolução horizontal de aproximadamente $270 \mathrm{~km}^{2}$ em uma grade global com espaçamento de $2,5^{\circ}$ x $2,5^{\circ}$ de latitude/longitude. Obtidos através do site National Oceanic and Atmospheric Admistration (NOAA). Uma descrição mais detalhada desse modelo pode ser encontrada em Kalnay et al. (1996).

As variáveis utilizadas foram: componente zonal e meridional do vento $\left(\mathrm{m} . \mathrm{s}^{-1}\right)$, temperatura $\left({ }^{\circ} \mathrm{C}\right)$, temperatura potencial, pressão $(\mathrm{hPa})$, geopotencial (mgp), umidade relativa (\%), movimento vertical $\left(\mathrm{hPa} . \mathrm{s}^{-1}\right)$ e vorticidade $\left(\mathrm{s}^{-1}\right)$. Para auxiliar nas análises foram calculadas variáveis atmosféricas derivadas como: divergência do vento $\left(\mathrm{m} . \mathrm{s}^{-1}\right)$ e umidade (m.g.kg $\left.{ }^{-1} \cdot \mathrm{s}^{-1}\right)$, espessura da camada, temperatura potencial equivalente, advecção de temperatura $\left({ }^{\circ} \mathrm{C} \cdot \mathrm{s}^{-1}\right)$. As análises foram realizadas para as variáveis em superfície, $925 \mathrm{hPa}, 850 \mathrm{hPa}, 700 \mathrm{hPa}, 500 \mathrm{hPa}$ e $200 \mathrm{hPa}$.

Foram empregadas radiossondagens para estações situadas no NEB, para o ponto mais próximo possível da localização da extremidade frontal e dos processos convectivos, sendo essas estações as seguintes: Caravelas BA, Salvador - BA, Petrolina - PE, Recife - PE e Natal - RN.

Essas radiossondagens foram obtidas do banco de dados do Centro de Previsões de Tempo e Estudos Climáticos (CPTEC) / Instituto Nacional de Pesquisas Espaciais (INPE), através do site http://bancodedados.cptec.inpe.br, já plotadas em diagramas Skew T - Log P, de acordo com os casos selecionados, dentro do período de estudo (20002009).

\subsection{Metodologia}

\subsubsection{Identificação e agrupamento dos casos.}

Inicialmente, através de uma análise das imagens de satélites, foram encontradas as bandas frontais no NEB de acordo com descrições apresentados em Fedorova et al. (2016). Na segunda etapa, todos eventos selecionados foram verificados e confirmados de acordo com a metodologia descrita no mesmo artigo. Ou seja, foram avaliadas a existência de:
1. cavado do ciclone barotrópico (pelo campo de pressão ao nível do mar) e vorticidade ciclônica (em $1000 \mathrm{hPa}$ );

2. zona com elevados gradientes de espessura na camada $1000 / 500 \mathrm{hPa}$;

3. zona com confluência de linhas de corrente em baixos níveis $(925$ e $850 \mathrm{hPa})$;

4. localização entre as regiões de advecção de temperatura e temperatura potencial equivalente positiva e negativa ( 925 e $850 \mathrm{hPa})$.

Os eventos selecionados foram separados e agrupados de acordo com as características de nebulosidade em dois grupos $\mathrm{G}$ :

Grupo 1 (G1): Atividades convectivas sobre o NEB, organizadas pela extremidade frontal.

Grupo 2 (G2): Banda de nebulosidade frontal pouco ativa sobre o NEB.

Através de uma análise criteriosa de todos os campos citados acima (báricos e térmicos) e ainda de umidade, os grupos G1 e G2 foram caracterizados da seguinte forma:

G1: Existência da zona frontal no NEB (pelos critérios citados acima) com atividade convectiva organizada na extremidade frontal sobre esta região.

G2: Existência da zona frontal no NEB (pelos critérios citados acima) sem atividade convectiva organizada no NEB.

Para estes grupos foi realizada uma divisão sazonal considerando-se verão austral (dezembro, janeiro, fevereiro); inverno austral (junho, julho, agosto); outono austral (maço, abril, maio) e primavera austral (setembro, outubro, novembro). Esta divisão foi feita devido à diferença no padrão de circulação, de acordo com a época do ano. Após, realizou-se uma comparação entre os casos do grupo 1 (G1) e do grupo 2 (G2), para determinar os fatores dinâmicos que favorecem ou não o desenvolvimento de atividades convectivas associadas à extremidade frontal.

\subsubsection{Análise Termodinâmica dos eventos}

Analisaram-se os perfis atmosféricos, os quais foram obtidos através das radiossondagens e plotados em diagramas Skew-T, para os casos pertencentes aos grupos G1 e G2. Foram utilizadas as radiossondagens para a estação mais próxima possível da região onde se observaram as atividades convectivas para os casos do G1. E para a estação mais próxima possível de onde se observou a atuação da extremidade frontal para os casos do G2. Analisaram-se os índices de instabilidade Convective Available Potencial Energy (CAPE), K, Total Totals (TT) e Lifted index LI (Fedorova et al., 2017), que sintetizam as características termodinâmicas para típicas situações convectivas, servindo, dessa maneira, para determinar o possível potencial convectivo da área em estudo.

Foi realizada uma comparação entre as os grupos G1 e G2 para a determinação da real importância das con- 
dições termodinâmicas para o desenvolvimento dos processos convectivos associados à extremidade frontal.

\section{Resultados e Discussão}

\subsection{Atuação dos sistemas frontais no NEB}

Durante o período de estudo selecionou-se 103 eventos em que as frentes se deslocaram para a região tropical, causando aumento de nebulosidade e/ou chuva sobre o NEB. Nos grupos G1 e G2, foram classificados 68 e 35 eventos, respectivamente.

A distribuição sazonal dos eventos, mostra que o NEB sofre influência de SF durante todo ano (Tabela 1). Porém, notou-se durante o período de estudo (2000-2009) que há uma significativa variação no número de SF que atuaram no NEB de um ano para outro. Esses resultados estão de acordo com os obtidos por Kousky (1979), que, após analisar dez anos de dados (1961-1970), chegou à conclusão de que as frentes atuam no sul da Bahia durante todo ano, com uma frequência maior de março a dezembro. Com base nos dados obtidos de Fedorova et al. (2016), os SF em Salvador nos anos 2004-2006 foram observados durante todo ano com um pequeno aumento de número entre abril e julho e em outubro.

A Tabela 1 também mostra a variação na quantidade de eventos de um ano para outro e a variação sazonal na distribuição dos casos. Em média houve a atuação de aproximadamente 10 frentes sobre o NEB. Sendo que os anos de 2000 com 19 casos, de 2002 com 14 casos e 2007 com 13 casos tiveram um número de casos acima da média. No entanto, os anos de 2004, com apenas 5 eventos observados, o ano de 2005 com 6 casos e o ano de 2008 onde 7 frentes atuaram no NEB, foram os anos que tiveram um número de ocorrência abaixo da média.
A variabilidade interanual no número de casos de $\mathrm{SF}$ que atuaram no NEB pode estar relacionada com ocorrências de eventos de El Niño e La Niña. Observou-se que o ano (2000) em que se notou um número maior de ocorrências de influências frontais no NEB foi um ano em que ocorreu uma La Niña. Da mesma forma, o ano com uma frequência menor no número de casos foi um ano (2004) de El Niño. Esses resultados estão de acordo com os encontrados por Cavalcanti (1996), que verificou que em anos de El Niño os SF em sua maioria não se deslocam de sul para norte e são intensificados na Região Sul. E com os resultados encontrados por Fedorova e Carvalho (2000) que verificaram que em anos de El Niño as frentes atuaram mais sobre o Rio grande do Sul e Uruguai.

\subsection{Variação sazonal das frentes no NEB}

$\mathrm{O}$ maior número de eventos em que SF influenciaram as condições do tempo no NEB ocorreu durante $o$ verão austral, com 33 ocorrências. Para as demais estações do ano, não houve uma variação significativa. Durante o outono austral, notaram-se 26 casos; no inverno austral foram registrados 23 e a primavera austral registrou 21 eventos.

Os resultados da análise da frequência das frentes com e sem convecção na sua extremidade será descrita a seguir. No inverno austral, 22 casos, dos 23 , pertencem ao G2 (sem convecção); nas outras estações do ano o número de eventos do G1 foi sempre superior aos do G2.

Foram observados eventos do G1 em todas as estações do ano, porém, durante o inverno austral, foi verificado apenas um caso pertencente a esse grupo. A frequência maior de ocorrências do GRUPO 1 ocorreu durante o verão austral. Para o GRUPO 2, a estação na qual se registrou uma frequência maior de eventos foi o inverno austral e não foram verificados casos do G2 du-

Tabela 1 - Quantidade dos casos de zonas frontais sobre o NEB, para o G1 e o G2 durante o período de estudo (2000-2009) e para as estações de transição, verão e inverno austral.

\begin{tabular}{|c|c|c|c|c|c|c|c|c|c|}
\hline \multirow{2}{*}{$\begin{array}{c}\text { Estações } \\
\text { Ano/Grupos }\end{array}$} & \multicolumn{2}{|c|}{ Verão Austral } & \multicolumn{2}{|c|}{ Transição Fria } & \multicolumn{2}{|c|}{ Inverno Austral } & \multicolumn{2}{|c|}{ Transição Quente } & \multirow[t]{2}{*}{ Total Anual } \\
\hline & G1 & $\mathrm{G} 2$ & G1 & $\mathrm{G} 2$ & G1 & $\mathrm{G} 2$ & G1 & $\mathrm{G} 2$ & \\
\hline 2000 & 5 & & 3 & 2 & & 6 & 2 & 1 & 19 \\
\hline 2001 & 4 & & & 1 & & 1 & 1 & & 7 \\
\hline 2002 & 7 & & 1 & & & 2 & 2 & 2 & 14 \\
\hline 2003 & 3 & & 3 & 1 & & 2 & 1 & 1 & 11 \\
\hline 2004 & 1 & & & & & 2 & 1 & 1 & 5 \\
\hline 2005 & 2 & & & & & 2 & 1 & 1 & 6 \\
\hline 2006 & 1 & & 6 & & & 2 & 1 & & 10 \\
\hline 2007 & 3 & & 2 & 3 & & 2 & 3 & & 13 \\
\hline 2008 & 4 & & & & 1 & 1 & & 1 & 7 \\
\hline 2009 & 3 & & 4 & & & 2 & 2 & & 11 \\
\hline Total por Grupo & 33 & & 19 & 7 & 1 & 22 & 14 & 7 & 103 \\
\hline Total por Estação & & & & & & & & & \\
\hline
\end{tabular}


rante o verão austral. Este fato está diretamente ligado à variação sazonal na dinâmica da circulação, pois, entre o final da estação de transição fria e o início da transição quente, há um resfriamento da superfície continental. A Alta Subtropical do Atlântico Sul (ASAS) se aproxima da América do Sul, inibindo a convecção profunda sobre o continente. Já entre o final da estação de transição quente e o início da transição fria, a superfície continental é aquecida, o que gera áreas de baixa pressão em superfície e divergência em altos níveis da troposfera, favorecendo o desenvolvimento de sistema convectivos.

\subsection{Ligação da zona frontal no NEB com onda de Rossby}

As zonas frontais no NEB apresentam parte da onda de Rossby sobre Atlântico Sul. Geralmente, os primeiros ciclones de onda são observados no sul do oceano Atlântico, perto do continente sul Africano e a zona frontal do último ciclone da onda atinge o NEB (Fig. 1). Foi constatado, tanto para os eventos pertencentes ao G1 como para os do G2, ligação entre a zona frontal analisada e restos frontais de uma onda anterior, ou interações com a onda frontal na sua retaguarda (Fig. 1). Essas ligações entre o sistema em análise e as ondas na sua vanguarda e na sua retaguarda intensificam a frente, sendo importante para que esta exerça influência sobre o NEB. Este resultado está em concordância com o encontrado nos estudos realizados por Cruz (2008), pois após analisar três anos de dados (2004-2006), concluiu que as interações entre uma onda frontal e restos deixados por uma onda anterior e/ou posterior eram importantes para a influência de SF em Alagoas.

\subsection{Padrões de circulação nos eventos com convecção organizada pela frente no NEB}

O desenvolvimento de convecção, organizada pela extremidade frontal, ocorreu quando foi observada confluência no escoamento em níveis baixos e médios, e forte difluência nos altos níveis da troposfera. Em baixos níveis, a confluência se origina da interação entre a circulação do ciclone ou de cavados nas proximidades do Sudeste do Brasil e da ASAS, (Figs. 2a e 2b, respectivamente). O padrão mostrado na Fig. 2a, foi observado em cerca de $46 \%$ dos casos, já o padrão da Fig. 2 b foi verificado em cerca de $54 \%$ dos casos. A diferença entre estes padrões é intensidade de cavado frontal.

No padrão (a) o ciclone baroclínico fica perto do Sudeste do Brasil e no padrão (b) o ciclone já localiza ao

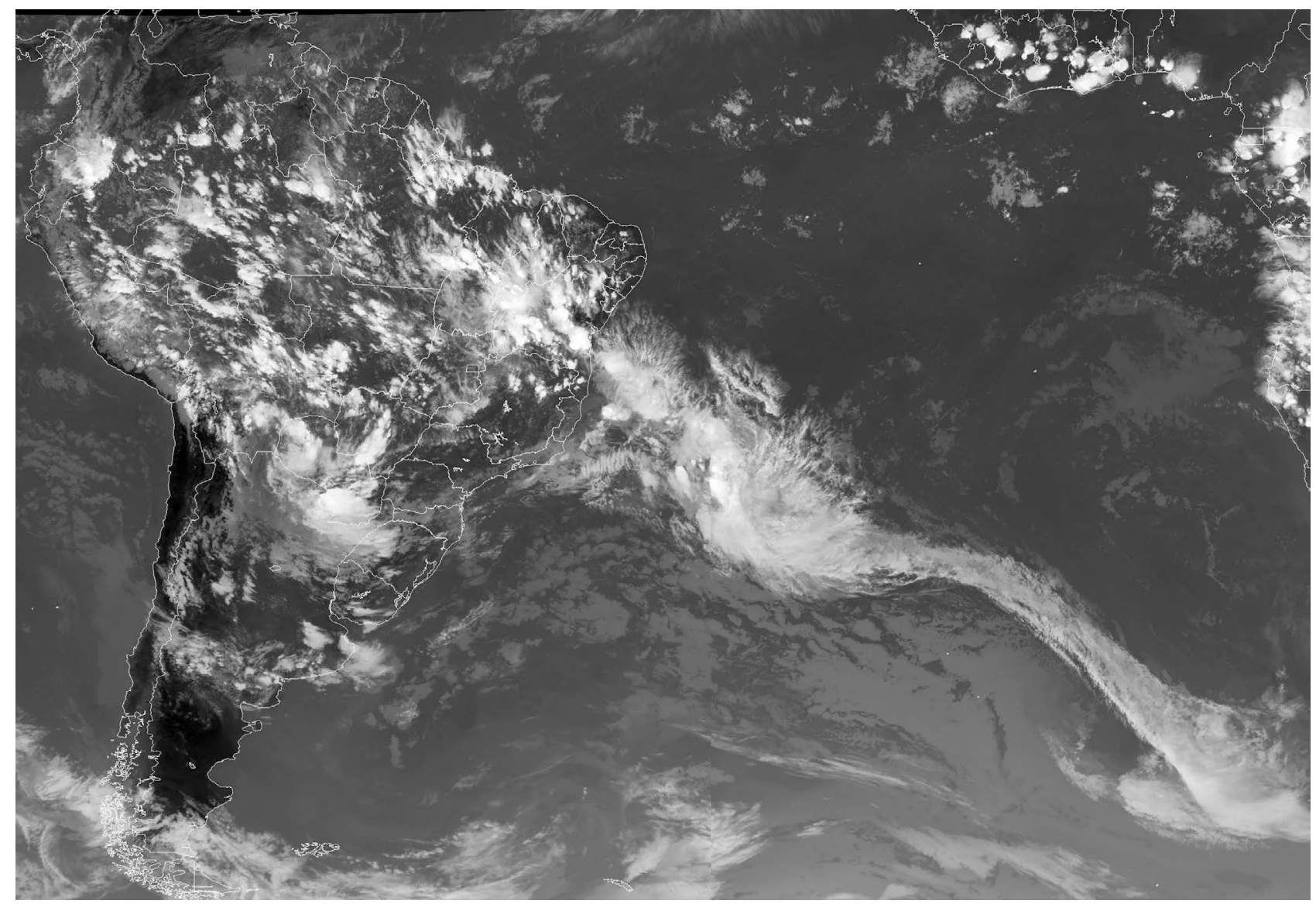

Figura 1 - Imagem de satélite do canal IR GOES 10.2-11.2 $\mu$ m e METEOSAT 10.8 $\mu \mathrm{m}$. 26/02/2008 18Z 

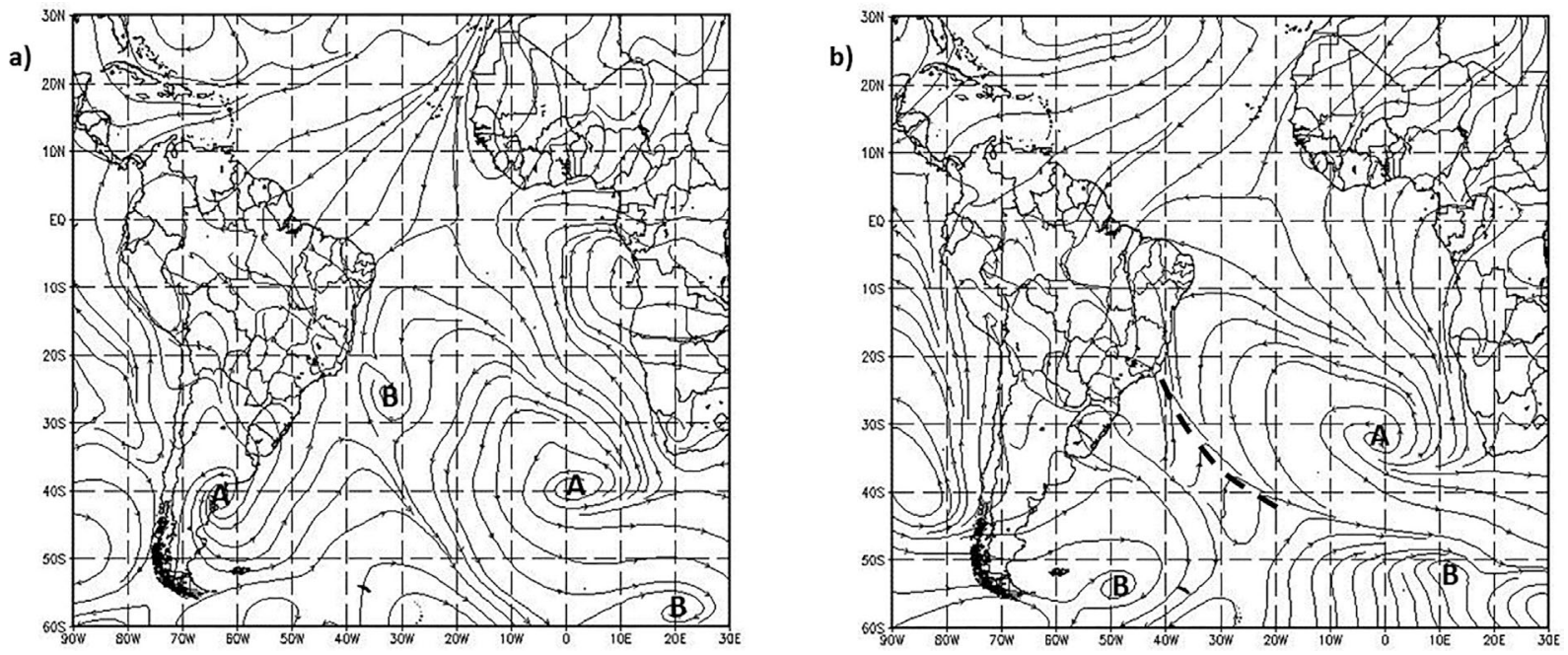

Figura 2 - Padrões de sistemas sinóticas em 925 hPa para formação frentes com atividade convectiva na sua extremidade no NEB em baixos níveis.

sul do continente Africano. Este mostra a diferença da posição da onda de Rossby, que foi citada no item 3.3.

A confluência em médios niveis origina-se pela convergência das correntes de ar do vórtice ciclônico (VC) sobre o oceano Atlântico (com centro próximo de $40^{\circ} \mathrm{S} /$ $40^{\circ} \mathrm{W}$ ) e anticiclone subtropical do Atlântico Sul (com centro próximo de $20^{\circ} \mathrm{S}, 10^{\circ} \mathrm{W}$ ) (Fig. 3a), sendo que esta configuração foi observada em $63 \%$ dos casos com convecção sobre o NEB. Em 17\% dos casos, principalmente entre a estação de transição quente $\mathrm{e} o$ início do verão Austral, foi observada uma variação do padrão anterior, onde o $\mathrm{VC}$ se encontra centrado em uma latitude mais alta, porém, nota-se que a confluência sobre o NEB é formada pela interação do anticiclone sobre o Atlântico e de cavado com eixo entre o Nordeste e o Sudeste do Brasil (Fig. 3b). A convecção sobre o NEB, organizada pela extremidade frontal, também foi favorecida, em $26 \%$ dos casos, pela presença de um cavado entre os anticiclones sobre o Atlântico e o interior do continente (Fig. 3c).
Em altos níveis, em 79\% dos eventos, a difluência das correntes de ar ocorreu entre a Alta da Bolívia e o VCAN (ou cavado) sobre o Oceano Atlântico (Fig. 4a). Tipicamente, nos eventos do G1, o VCAN estava posicionado em torno de $10^{\circ} \mathrm{S} / 20^{\circ} \mathrm{W}$ ), já o cavado tende a ficar um pouco mais afastado do continente. Dessa forma, a periferia oeste destes sistemas contribui para a difluência. Em $18 \%$ dos casos com convecção sobre o Nordeste, organizada pela extremidade frontal, foi observada a presença de um amplo cavado entre o Nordeste e o Sudeste do Brasil (Fig. 4b). Este padrão ocorreu, principalmente, nas estações de transição. A interação entre este cavado, a $\mathrm{AB}$ e o anticiclone sobre o Atlântico, também gera áreas de difluência sobre o a NEB, favorecendo o desenvolvimento convectivo sobre a Região. Além disso, conforme mostrado por Cruz (2008), a amplificação do cavado em altitude também favorece a incursão dos SF para latitudes mais baixas.
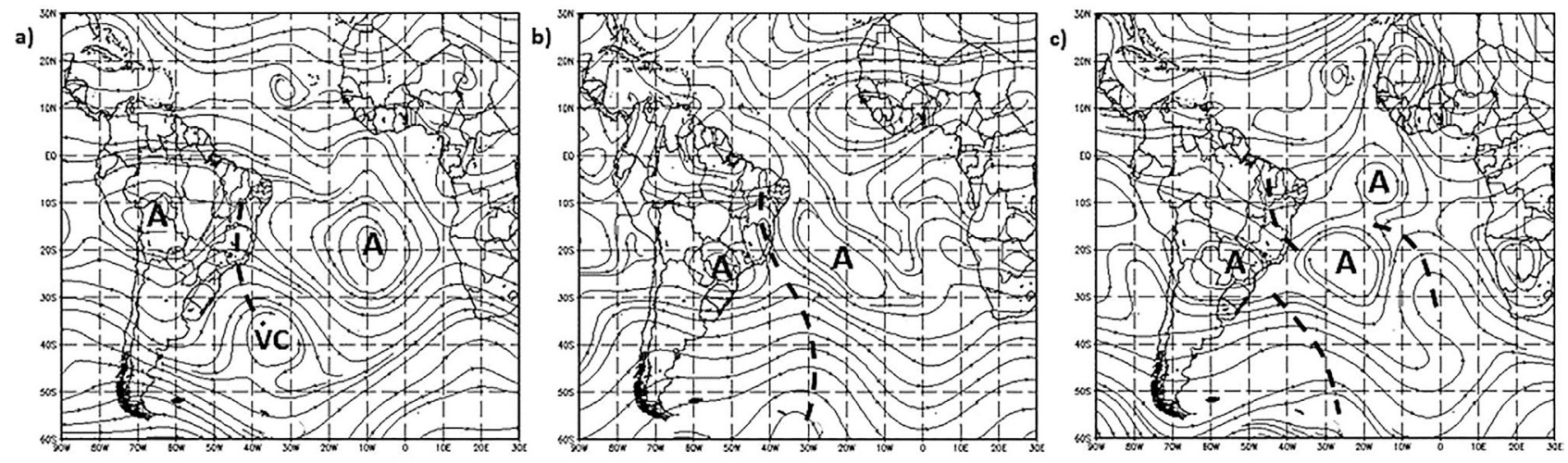

Figura 3 - Padrões típicos em médios níveis atmosféricos para os eventos em que se observou atividades convectivas na extremidade frontal sobre o $\mathrm{NEB}$, linhas de corrente em $500 \mathrm{hPa}$. 

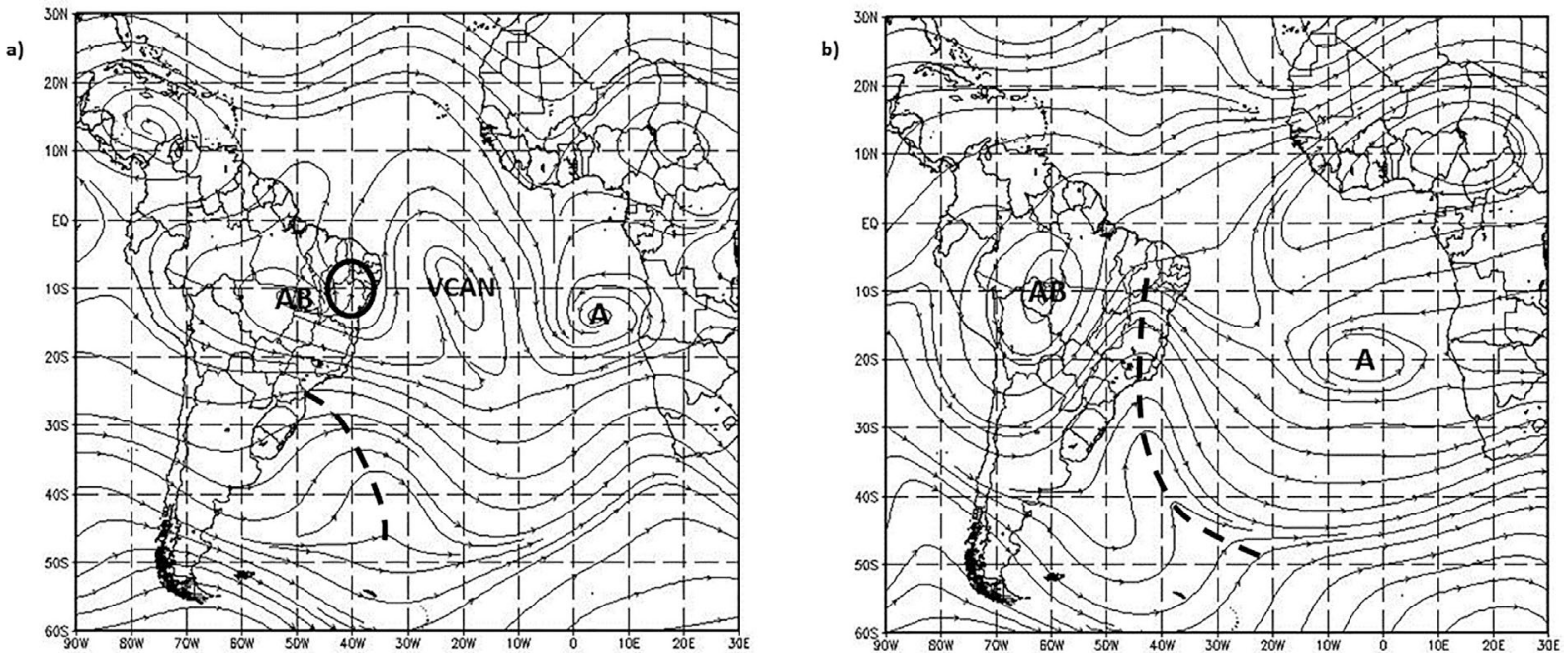

Figura 4 - Padrões típicos em altos níveis atmosféricos para os eventos em que se observou atividades convectivas na extremidade frontal sobre o NEB, linhas de corrente em $200 \mathrm{hPa}$.

\subsection{Padrões de circulação nos eventos sem convecção organizada pela frente no NEB}

A principal diferença entre os eventos com e sem desenvolvimento de convecção profunda sobre o NEB é a ausência de confluência em baixos níveis (Fig. 5). Nos eventos sem convecção, nota-se que o anticiclone pósfrontal se posiciona mais próximo do continente e mais ao norte em relação aos casos do G1, com centro variando entre $25-35^{\circ} \mathrm{S} / 40-45^{\circ} \mathrm{W}$. As correntes de ar deste anticiclone influenciam diretamente as condições de tempo no NEB, tipicamente com o escoamento de sudeste/leste favorecendo a formação de nebulosidade estratiforme e chuva em algumas áreas.

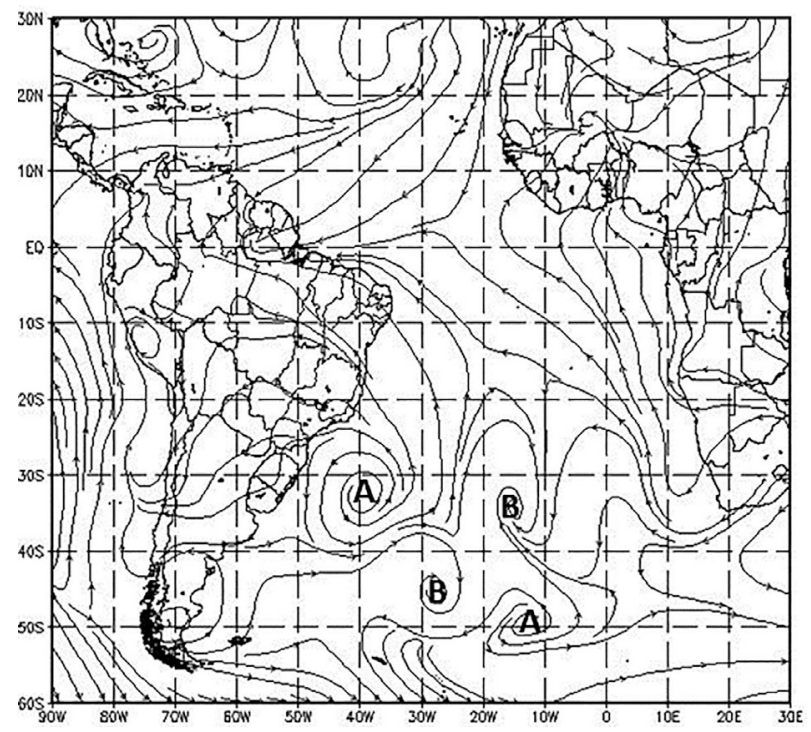

Figura 5 - Padrão em baixos (925 hPa) níveis para os eventos em que não se observou atividades convectivas na extremidade frontal sobre NEB.
Em níveis médios, na maioria dos casos em que a extremidade frontal não organizou convecção sobre o NEB, foi observada a influência direta de uma circulação anticiclônica centrada sobre o NEB (com centro, aproximadamente, em $20^{\circ} \mathrm{S} / 50^{\circ} \mathrm{W}$ ), dominando a circulação em grande parte do centro-norte do continente (Fig. 6a). Em alguns casos também se observou que o anticiclone esteve posicionado sobre o centro-sul do continente e observouse a presença de um cavado sobre o Atlântico. A presença simultânea desses dois sistemas gera uma região de difluência no escoamento sobre o NEB (Fig. 6b), o que não favorece o desenvolvimento de sistemas convectivos sobre esta Região.

Em altos níveis, a principal diferença dos casos G2 do G1 é a ausência do VCAN do tipo clássico ou cavado intenso, nas proximidades do NEB. A corrente zonal com fracos cavados sobre o NEB predomina em altos níveis nos casos sem convecção (Fig. 7a). Em alguns casos, observou-se a presença de um cavado sobre o NEB (Fig. 7b), porém, se comparado aos casos do G1, nota-se que tanto o cavado quanto o anticiclone estão deslocados mais para oeste; o que faz com a região de difluência fique principalmente sobre o Norte do Brasil.

\subsection{Considerações sobre os fatores termodinâmicos na formação dos processos convectivos sobre o NEB}

Comparando os perfis verticais da atmosfera para os eventos do G1 (com convecção), e para os eventos do G2 (sem convecção); observou-se, tanto para os eventos do G1 quanto para os do G2, que a atmosfera se apresentava condicionalmente instável.

Tanto para os eventos do G1 quanto para os do G2 os níveis baixos da atmosfera apresentaram umidade elevada. Contudo, os perfis analisados para os casos do G2 apresentavam uma camada relativamente mais seca entre 

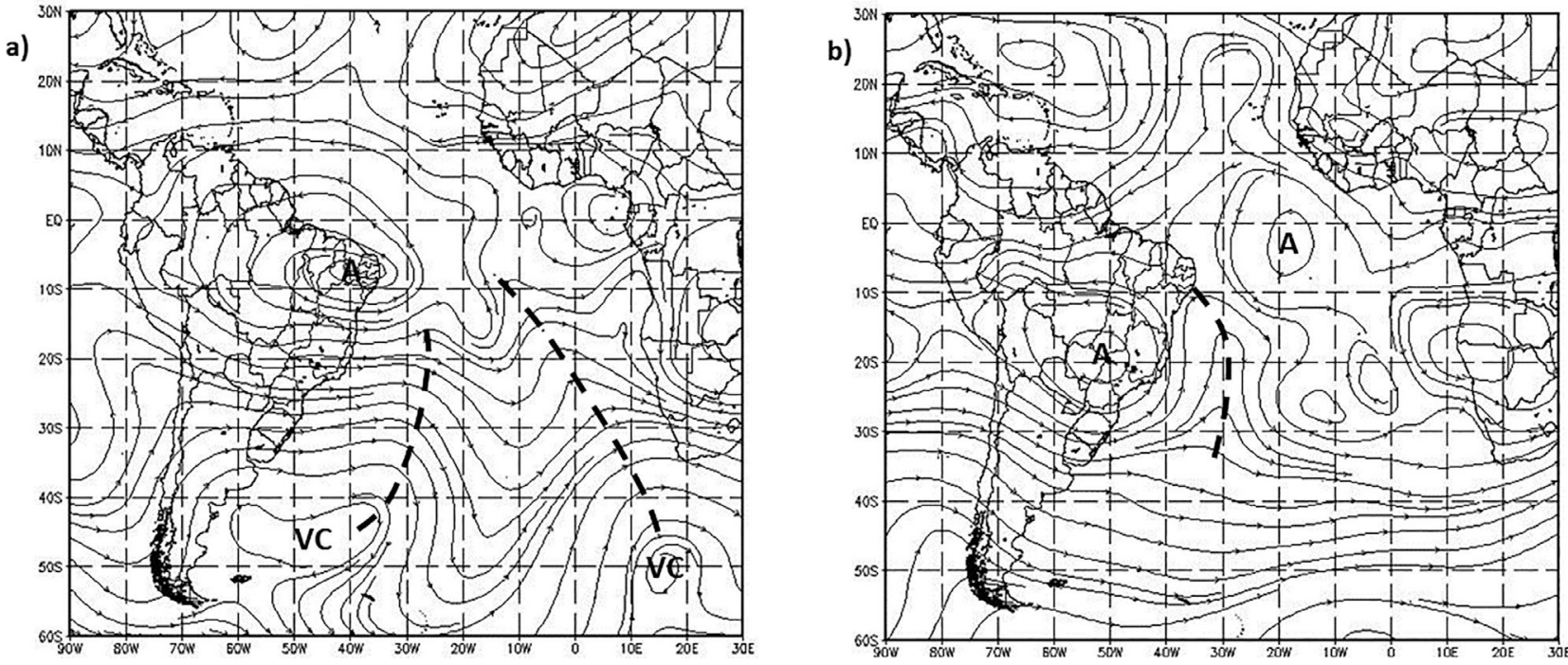

Figura 6 - Padrões típicos em médios níveis atmosféricos para os eventos em que não se observou atividades convectivas na extremidade frontal sobre $\mathrm{NEB}$, linhas de corrente em $500 \mathrm{hPa}$.
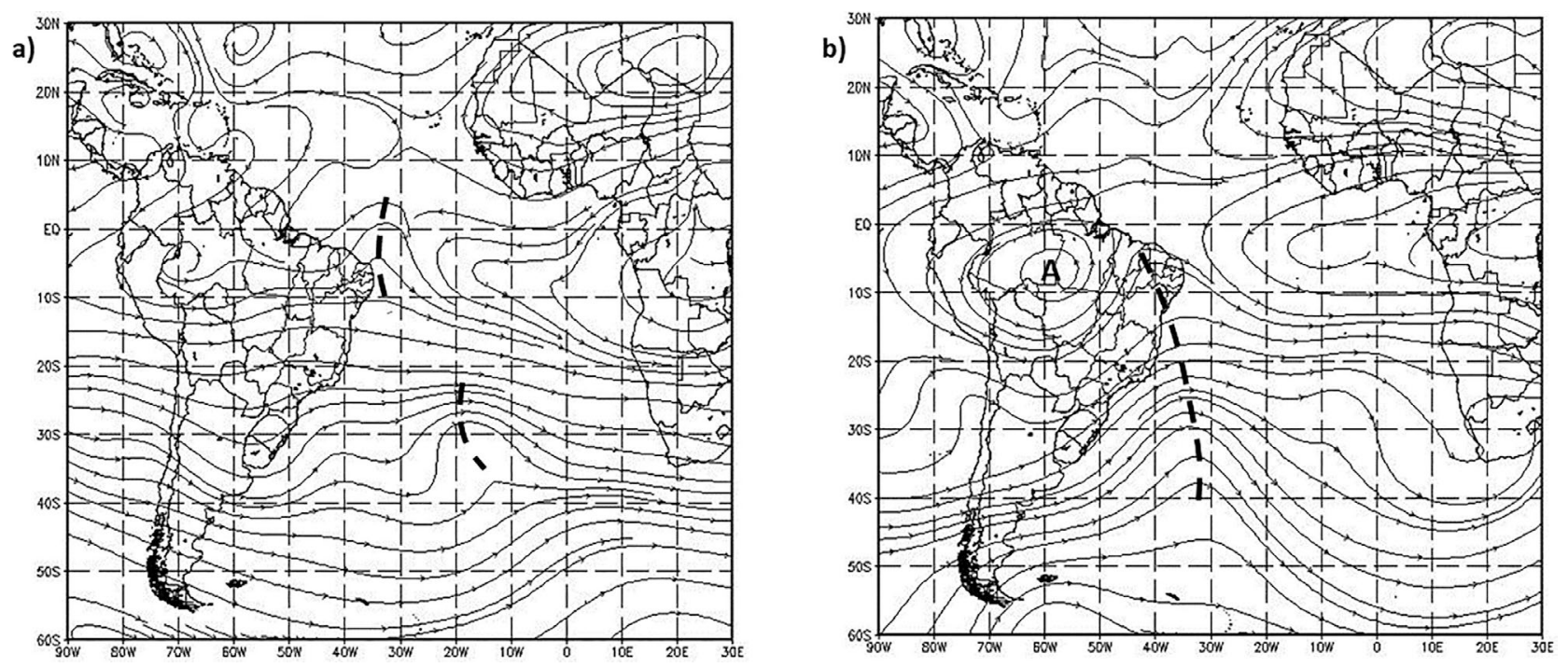

Figura 7 - Padrões típicos em altos níveis atmosféricos para os eventos em não que se observaram atividades convectivas na extremidade frontal sobre $\mathrm{NEB}$, linhas de corrente em $200 \mathrm{hPa}$.

$700 \mathrm{hPa}$ e $500 \mathrm{hPa}$, o que, provavelmente, está relacionado com a diferença no padrão de circulação e a sazonalidade dos eventos estudados.

O CAPE e o Índice de Levantamento (LI), apresentou valores que denotam a possibilidade de formação de processos convectivos em $88 \%$ e $85 \%$ dos casos do G1, e $63 \%$ e $75 \%$ dos casos do G2, respectivamente. Para o total de eventos analisados, os índices $\mathrm{K}$ e Total-Totals (TT) não se mostraram eficientes para previsão de eventos convectivos sobre o NEB; pois, em apenas $39 \%$ e $15 \%$ dos casos do G1 (com convecção), respectivamente, indicaram valores consistentes com as condições de tempo e de nebulosidade observadas.

\section{Conclusões}

Para utilizar os resultados do estudo na prática operacional de previsão de tempo em curto prazo, foram criados os padrões de sistemas sinóticos associados com eventos de desenvolvimento de convecção profunda na extremidade frontal e sem esta convecção.

Ficou evidente que o NEB sofre influência de SF durante todas as estações do ano. No entanto, dependendo da época do ano, as zonas frontais influenciam o tempo sobre o NEB de maneira distinta. Em 22 dos 23 casos, ocorridos durante o inverno austral e para a grande maioria dos casos do início da estação de transição fria (19 
dos 26 casos encontrados nesta estação) e final da primavera do hemisfério sul ( 7 dos 21 casos encontrados nesta estação), observou-se que a nebulosidade da extremidade frontal sobre o NEB foi pouco ativa (G2). Durante esse período do ano, a atuação das zonas frontais no NEB restringiu-se com maior frequência ao estado da Bahia, principalmente na parte sul. Sendo encontrados eventos com essa característica em 35 casos do total analisado.

Nos casos verificados durante o verão austral (33), e para os casos ocorridos no início da estação de transição fria ( 7 dos 26 casos desta estação) e final da primavera no hemisfério sul (14 dos 21 casos desta estação), evidenciou-se a intensificação da extremidade sobre o NEB. Dessa forma, organizaram-se fortes processos convectivos sobre grande parte do NEB, configurando eventos pertencentes ao G1, que foram encontrados em 68 casos do total analisados.

Concluiu-se, finalmente, que nos eventos com convecção intensa, organizada pela extremidade frontal sobre o NEB, os sistemas sinóticos geram uma região de confluência no escoamento em baixos e médios níveis sobre o NEB. Entretanto, nas situações sem convecção organizada, observa-se um padrão de circulação anticiclônica. Em altos níveis a presença do VCAN do tipo clássico ou de um cavado intenso sobre o Atlântico Tropical Sul, nas proximidades do NEB, é um fator determinante para o desenvolvimento de convecção organizada pela extremidade frontal.

A análise termodinâmica para os casos pertencentes aos grupos 1 e 2 mostrou que os índices de instabilidade analisados (CAPE, K, TT, LI) não mostraram comportamentos muito distintos, quando comparados os valores encontrados para os casos do G1 e G2. Os índices K e TT mostraram-se não eficientes para previsão de eventos convectivos sobre o NEB. Os índices também, muitas vezes, não denotaram as condições reais de nebulosidade observadas através das imagens de satélite.

Por isso, pode-se determinar que a previsão de processos convectivos sobre o NEB, associados à extremidade frontal, não pode ser realizada somente por meio da análise de perfis termodinâmicos e de índices de instabilidade. Pois os índices termodinâmicos mostram potencial para o desenvolvimento convectivo em grande parte dos casos estudados, tanto para o G1 quanto para o G2. CAPE e LI têm os melhores resultados, mas as diferenças entre G1 e G2 não são muito significativas. O potencial convectivo dado pelos índices termodinâmicos não é suficiente para o desenvolvimento dos processos convectivos, sendo necessária a presença de mecanismos dinâmicos para início desses processos.

\section{Agradecimentos}

Os autores agradecem a Coordenação de Aperfeiçoamento de Pessoal de Nível Superior (CAPES) pelo apoio financeiro concedido durante e para a realização deste estudo.

\section{Referências}

BRABO ALVES, J.M.; SILVA, E.M.; SOMBRA, S.S.; BARBOSA, A.C.B.; SANTOS, A.C.S., LIRA, M.A.T. Eventos Extremos Diários de Chuva no Nordeste do Brasil e Características Atmosféricas. Revista Brasileira de Meteorologia, v. 32, n.2, p. 227-233, 2017.

CAVALCANTI, I.F.A.; KOUSKY, V.E. Tempo e Clima no Brasil. São Paulo: Ed. Oficina de textos, v. 1, p. 135, 2009.

CAVALCANTI, I.F.A.; KOUSKY, V.E. Climatology of outh american cold fronts. In: Proc. $7^{\circ}$ Internatinal Conference On Southern Hemisphere Meteorology And Oceanograpy. New Zealand: American Meteorological Society, CD-Rom, 2003.

CRUZ, C.D. Determinação Sinótica dos Fatores que Favorecem As Influências Frontais Sobre O Estado De Alagoas. M.Sc. Thesis, Federal University of Alagoas, Maceió, 2008.

FEDOROVA, N.; LEVIT, V.; CRUZ C.D. On frontal zone analysis in the tropical region of the Northeast Brazil. Pure and Applied Geophysics, v. 173, p. 1403-1421, 2016.

FEDOROVA, N.; CARVALHO, M.H. Processos sinóticos em anos de La Niña e de El Niño. Parte II: Zonas frontais. Revista Brasileira de Meteorologia, v. 15, n. 2, p. 57-72, 2000.

FEDOROVA, N.; PONTES DA SILVA, B.F.; LEVIT, V. 2017. Análise e previsão prática das nuvens pelo diagrama termodinâmico SKEW-T/LOG-P, 227 p., 2017.

GEMIACKI, L. Atuação de Sistemas Frontais na Estação Seca do Nordeste do Brasil. M.Sc. Thesis, Federal University of Alagoas, Maceio, 2005.

JUSTI, M.G.A.; SILVA DIAS, M.A.F. A frequência de fenômenos meteorológicos na américa do sul: uma climatologia. Anais do XI Congresso Brasileiro de Meteorologia, Foz do Iguaçu, CD-Rom, 2002.

KALNAY , E. The NCEP/NCAR 40-year reanalysis project. Bull. Amer. Meteor. Soc., vol. 77, n.3, p 437-470, 1996.

KOUSKY, V.E., Frontal Influences on Northeast Brazil. Monthly Weather Review, v. 107, n. 9, p. 1140-1153, 1979.

LEMOS, C.F. \& DE CALBETE, N.O., Sistemas frontais que atuaram no litoral de 1987 a 1995. In: Climanálise Especial Edição Comemorativa de 10 Anos. Brasília, MCT/ INPE/CPTEC, 1996.

OLIVEIRA, A.S. Interações Ente Sistemas Frontais na América do Sul e a Convecção da Amazônia. Dissertação de Mestrado, INPE, São José dos Campos, São Paulo, 1996.

SINHORI, N.P. Estrutura Vertical Das Frentes Frias Durante A Passagem Do Sul Até O Nordeste Brasileiro, Entre 2000 E 2012. Dissertação de Mestrado, Instituto de Ciências Atmosféricas, Universidade Federal de Alagoas, Maceió, 198 p., 2015.

\section{Endereços de Internet}

National Center for Environmental Prediction (NCEP) / National Center for Atmospheric Research (NCAR) - http://www. cdc.noaa.gov.

National Oceanic and Atmospheric Admistration (NOAA) http://www.ncdc.noaa.gov/gibbs.

License information: This is an open-access article distributed under the terms of the Creative Commons Attribution License (type CC-BY), which permits unrestricted use, distribution and reproduction in any medium, provided the original article is properly cited. 